\title{
Extracorporeal shockwave therapy for patellar tendinopathy: a review of the literature
}

\author{
M T van Leeuwen, J Zwerver, I van den Akker-Scheek
}

Center for Sports Medicine, University Center for Sport, Exercise and Health, University Medical Center Groningen, University of Groningen, Groningen, The Netherlands

Correspondence to:

J Zwerver, Center for Sports

Medicine, UMC Groningen, P.O

Box 30.001, 9700 RB Groningen,

The Netherlands; j.zwerver@ sport.umcg.nl

Accepted 24 July 2008 Published Online First

21 August 2008

\begin{abstract}
Background and purpose: Extracorporeal shockwave therapy (EWST) has become a popular treatment for patellar tendinopathy. The purpose of this review was to study the effectiveness of ESWT treatment for patellar tendinopathy; to draft guidelines for an effective treatment protocol of ESWT treatment; and to identify topics for further research.
\end{abstract}

Methods: A computerised search of the Medline and Embase databases was conducted on 1 August 2007, to identify studies dealing with the effectiveness of ESWT for patellar tendinopathy.

Results: Seven articles describing the effectiveness of ESWT on patellar tendinopathy, all published after 2000, were included. These studies included a total of 283 patients (298 tendons), 204 of whom (215 tendons) were assigned to ESWT treatment. The treatment results were positive but most studies had methodological deficiencies, small numbers and/or short follow-up periods. Method of application and shockwave generation, energy level, number and frequency of treatments, use of (local) anaesthesia and method of localisation were variable. Conclusion: ESWT seems to be a safe and promising treatment for patellar tendinopathy with a positive effect on pain and function. Based on current knowledge it is impossible to recommend a specific treatment protocol. Further basic and clinical research into the working mechanism and effectiveness of ESWT for patellar tendinopathy are necessary.

Patellar tendinopathy, also called jumper's knee, is a chronic overuse injury of the patellar tendon causing pain at the inferior pole of the patella. ${ }^{1}$ The prevalence is particularly high in athletes. ${ }^{2}{ }^{3}$ Among elite volleyball and basketball players a prevalence over $40 \%$ has been described. ${ }^{4}$ Because of its chronicity and a lack of consensus about which treatment is the most adequate, ${ }^{45}$ patellar tendinopathy has a major impact on the career of many athletes and for some it is the reason to end their career prematurely. ${ }^{267}$

The aetiology of patellar tendinopathy is not completely understood, but repetitive overload is thought to be an important factor. ${ }^{89}$ Histopathological examinations of affected patellar tendons revealed the absence of inflammatory cells and no increased levels of prostaglandin. ${ }^{8}$ Consequently, the former term tendinitis should be replaced by tendinosis, which reflects the underlying process of tendon degeneration and the failed healing response. ${ }^{10}$ The term tendinopathy is now often used clinically. ${ }^{10}$

Based on the present literature it is not possible to decide what is the most appropriate and effective treatment for patellar tendinopathy. ${ }^{11} 12$
The results of the many conservative treatments are not always consistent or evidence-based. ${ }^{7} 8$ 13-15 Eccentric training has been proposed as the best conservative treatment, but results are not convincing. 'When conservative treatment fails, most patients proceed to surgical treatment, ${ }^{11}{ }^{12}$ which according to Bahr et al produces excellent or good results in at most $45 \%$ of patients. ${ }^{4}$

Since the early 1990s, extracorporeal shockwave therapy (ESWT) has been used for the treatment of several chronic tendinopathies. ${ }^{16}$ This non-invasive and safe therapy originates from urology, where it is used to pulverise kidney stones (lithotripsy). ${ }^{17}$ In some studies, ESWT has shown promising results in the treatment of rotator cuff tendinopathy, extensor tendinopathy of the elbow and chronic plantar fasciitis. ${ }^{16-20}$ ESWT is nowadays used as a treatment for patellar tendinopathy as well. The underlying working mechanism of ESWT on tendinopathies is not completely understood, though. Both an analgesic effect and a stimulating effect on tissue regeneration have been suggested as possible working mechanisms. ${ }^{20-22}$ There is no consensus about ESWT treatment protocols. ${ }^{16}$ Controversy exists about method of application and shockwave generation (focused or radial), energy level to be used, number and frequency of treatments, use of (local) anaesthesia and method of localisation (see table 1$).{ }^{16} 20$

The main purpose of this review was to study the effectiveness of ESWT treatment for patellar tendinopathy. The ultimate goal is to draft guidelines for an effective treatment protocol and to identify topics for further research.

\section{MATERIALS AND METHODS}

\section{Literature search}

A computerised search of the Medline and Embase databases was conducted on 1 August 2007 (for search terms see fig 1). The search was limited to literature published in English or German. There was no limitation for publication year. Animal studies were excluded. Articles were defined to be relevant for this review if their subject was ESWT for patellar tendinopathy. Further, all reference lists were hand-searched for other relevant articles. The selected articles were reviewed by the authors and judged on their relevance and contribution to the subject of this study. All seven studies published about ESWT for patellar tendinopathy were included. All authors independently assessed the methodological quality of these seven studies using the Delphi criteria. ${ }^{23}$ Keywords were: patellar tendinopathy, jumper's knee, shockwave therapy, treatment, review. 
Table 1 Variables of influence on the effectiveness of ESWT

\begin{tabular}{|c|c|}
\hline Variables & Details \\
\hline $\begin{array}{l}\text { Shockwave } \\
\text { generation: }^{16} 17192029\end{array}$ & Site of most effects: \\
\hline - Focused shockwave & > Depth \\
\hline $\begin{array}{l}\text { Radial pressure wave } \\
\text { Energy level }\left(\mathrm{mJ} / \mathrm{mm}^{2}\right)^{16}:^{29}\end{array}$ & $\begin{array}{l}\text { Superficial } \\
\text { With an energy level of }>0.6 \mathrm{~mJ} / \mathrm{mm}^{2} \text { therapy will } \\
\text { be painful and macroscopic lesions will appear. }{ }^{16}\end{array}$ \\
\hline \multicolumn{2}{|l|}{$\rightarrow \operatorname{low}(<0.08)$} \\
\hline \multicolumn{2}{|l|}{$\checkmark$ medium $(0.08-0.28)$} \\
\hline \multicolumn{2}{|l|}{$>\operatorname{high}(>0.6)$} \\
\hline $\begin{array}{l}\text { Number of treatments, time } \\
\text { interval between treatments and } \\
\text { shockwave frequency }\end{array}$ & $\begin{array}{l}\text { If there is more than one treatment, effects will be } \\
\text { cumulative. }{ }^{13}\end{array}$ \\
\hline $\begin{array}{l}\text { Whether or not anaesthetics are } \\
\text { used. }\end{array}$ & $\begin{array}{l}\text { When using anaesthetics, a larger energy level can } \\
\text { be used. }{ }^{16}\end{array}$ \\
\hline \multicolumn{2}{|c|}{ Method of localisation: ${ }^{20}$} \\
\hline$\rightarrow$ anatomical & $\begin{array}{l}\text { Can be difficult with obese patients or when the } \\
\text { anatomy is disrupted as a result of surgery. }{ }^{20}\end{array}$ \\
\hline$\rightarrow$ image-guided focusing & $\begin{array}{l}\text { Very specific method; however, the site of the } \\
\text { lesion is not always consistent with the site where } \\
\text { the most pain is experienced. }{ }^{20}\end{array}$ \\
\hline$\checkmark$ clinical & $\begin{array}{l}\text { Shockwaves are applied directly to the site where } \\
\text { the most pain is experienced. This method of } \\
\text { localisation is not possible when using a local } \\
\text { anaesthetic. }^{20}\end{array}$ \\
\hline
\end{tabular}

\section{Results}

We found seven articles in which the effectiveness of ESWT on patellar tendinopathy was described, all published after 2000. These studies included a total of 283 patients (298 tendons), 204 of whom (215 tendons) were assigned to ESWT treatment. The main study characteristics are presented in table 2.

\section{Description of the studies}

In 2000 Vara et al studied the effectiveness of different ESWT energy levels for patellar tendinopathy in 27 patients. In this prospective, randomised, single-blind trial, one group received from one to five treatments with an energy level of 0.105 $0.437 \mathrm{~mJ} / \mathrm{mm}^{2}$. Another group (placebo) received one treatment with an energy level of $0.04 \mathrm{~mJ} / \mathrm{mm}^{2}$. Evaluation 2 years after the last treatment showed an improvement of $61 \%$ in the study group for VAS score, pressure pain and functional improvement. In the placebo group there was $7.5 \%$ improvement, and $74 \%$ and $15 \%$ of the patients in the study group and placebo group, respectively, thought the results were good or satisfactory. A limitation of this study was that it was not a double-blind trial, so patients knew whether they received placebo treatment or real treatment. This study showed good results in favour of the group that received ESWT treatment with an energy level of $0.105-0.437 \mathrm{~mJ} / \mathrm{mm}^{2}$, but the results could be biased, as a single-blind protocol was used. ${ }^{24}$

In a non-randomised, prospective pilot study in 2002 by Lohrer et al the effectiveness of radial shockwave therapy (RSWT) on patellar tendinopathy was evaluated in 45 patients who had not responded successfully to previous conservative treatments. Treatment consisted of from three to five RSWT sessions with an energy level from 0.06 to $0.18 \mathrm{~mJ} / \mathrm{mm}^{2}$. Pain at rest, pain during exercise, pressure pain (all VAS score) and painfree running time ( $\mathrm{min}$ ) were evaluated after 1, 4, 12, 26 and 52 weeks. During 1 year all scores improved significantly. One year after the last treatment $40 \%$ of the patients with patellar tendinopathy were pain-free, $24.4 \%$ had improved, and $36.5 \%$ of the patients showed no improvement. Although Lohrer et al did not include a control group for comparison purposes, they concluded that ESWT does have a therapeutic effect on patellar tendinopathy. ${ }^{25}$

In 2003 Peers et al retrospectively compared results of surgical treatment with ESWT for patellar tendinopathy in 27 patients (28 tendons). None of the patients had shown improvement after previous conservative treatment. After three ESWT sessions of $0.08 \mathrm{~mJ} / \mathrm{mm}^{2}$ in 14 patients ( $15 \mathrm{knees}$ ) and after tenotomy of the patellar tendon with resection of degenerative tissue in 13 patients, VAS and VISA scores, Roles \& Maudsley (R\&M) classification, length of the rehabilitation period and absence from work were evaluated. Two years after treatment no significant difference in VAS and VISA scores or R\&M classification was found, but the surgical treatment group did have a longer absence from work period postsurgically. A limitation of this study was lack of randomisation, so a selection bias may have possibly influenced the results. For patients it is difficult to choose between surgery and undergoing ESWT. The risk of complications, intensive postoperative rehabilitation and a considerable period of sick leave has to be considered. These factors may have influenced results in favour of the ESWT group. After this study, Peers et al concluded that ESWT is a good alternative to surgical treatment when conservative treatment fails in chronic patellar tendinopathy. ${ }^{6}$

In 2003 Peers wrote a thesis about the effectiveness of ESWT in Achilles and patellar tendinopathy. In a randomised clinical trial he compared focused ESWT treatment, consisting of three sessions with an energy level of $0.2 \mathrm{~mJ} / \mathrm{mm}^{2}$, with a placebo treatment (three sessions with an energy level of $0.03 \mathrm{~mJ} /$ $\mathrm{mm}^{2}$ ). The study group consisted of 21 patients; the control group included 20 patients. Evaluation after 12 weeks was performed using VISA score, R\&M classification and degree of functional impairment. There was a significant improvement in pain and function after ESWT treatment of $0.2 \mathrm{~mJ} / \mathrm{mm}^{2}$. Another evaluation variable was the VAS score, but for this variable no significant difference was found. In this study the follow-up lasted only 3 months, so no conclusion could be drawn for the long-term benefit. Another limitation of this study was that no limits were set on the severity of patellar tendinopathy at inclusion, so patients with mild or severe complaints were both included in this study. One might argue

\section{Figure 1 Literature search}

Results of search strategy in Medline for: (shock[TI] AND wave[TI]) OR shockwave[TI] OR eswt[TI]) AND treatment AND "review"[Filter]:

Results of search strategy in Medline for: (jumper's knee OR patellar tendinopathy $O R$ (patella* AND tendinopath*) OR (jumper* knee)) AND (extracorporeal shockwave OR eswt OR rswt OR shockwave OR shock wave):

$$
\downarrow n=207
$$

Results of search strategy in Embase for: ('patella tendinopathy' OR 'jumpers knee' OR 'patellar tendinopathy' OR (patella* AND tendinopath*) OR (jumper* AND 'knee'/syn)) AND ('extracorporeal shockwave' OR eswt OR shock wave OR 'shock wave'/syn OR 'extracorporeal lithotripsy'/syn):

Results of search strategy in Embase for: ('patella tendinopathy' OR 'jumpers knee' OR 'patellar tendinopathy' OR (patella* AND tendinopath*) OR (jumper* AND 'knee'/syn)) AND ('extracorporeal shockwave' OR eswt OR shockwave OR 'shock wave'):

$$
\downarrow n=23
$$

Total studies found after literature search

$$
\downarrow n=230
$$

Studies included after reading the title and abstract (from the total of 230 studies)

$$
\downarrow n=20 \text { (of which five with the subject ESWT in patellar tendinopathy) }
$$

Results after hand-search in reference lists for relevant articles (from the 20 included studies)

$\downarrow n=13$ (of which two with subject ESWT in patellar tendinopathy) Total studies included

$\mathrm{n}=33$ (of which seven with the subject ESWT in patellar tendinopathy) 


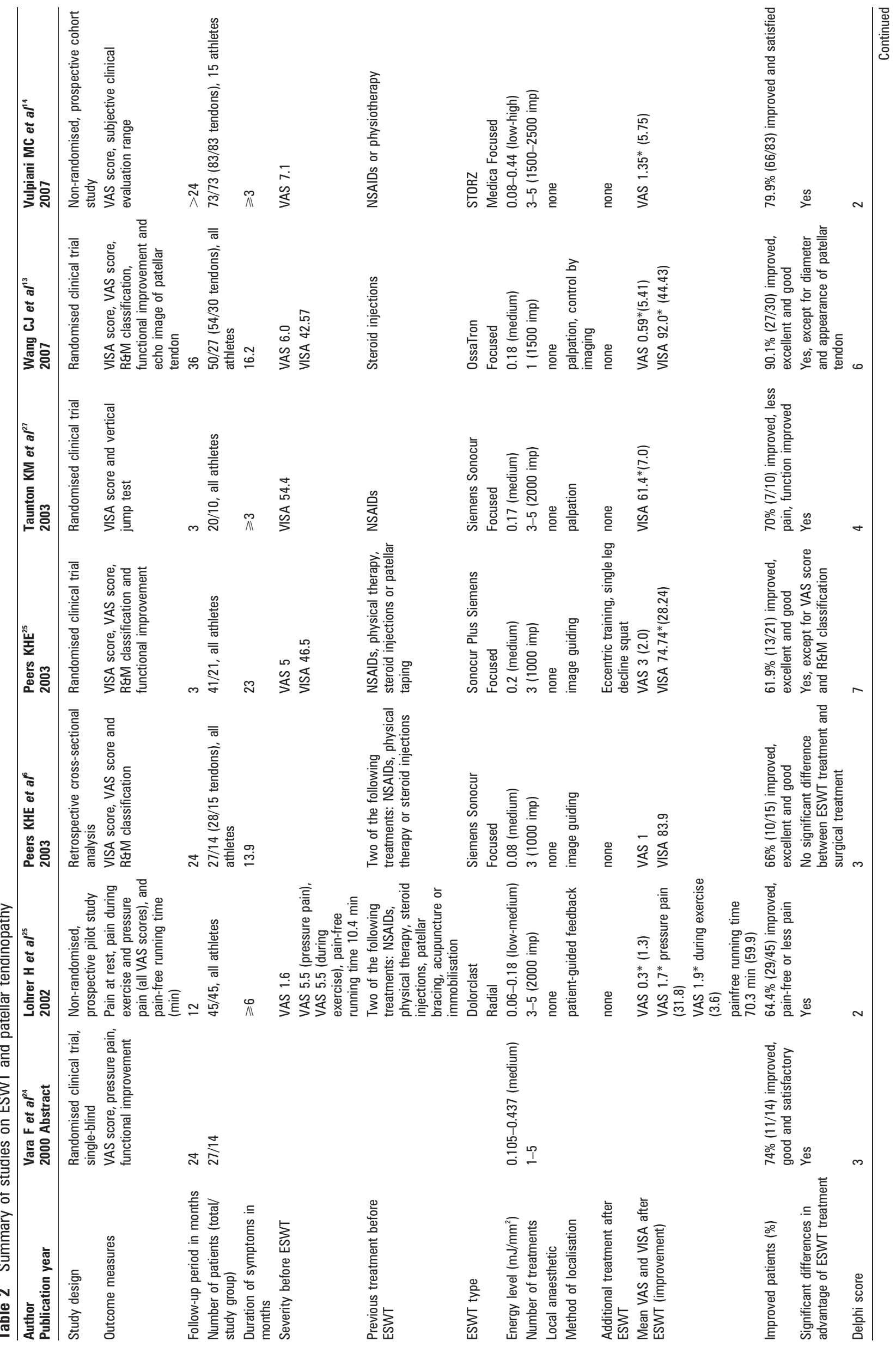




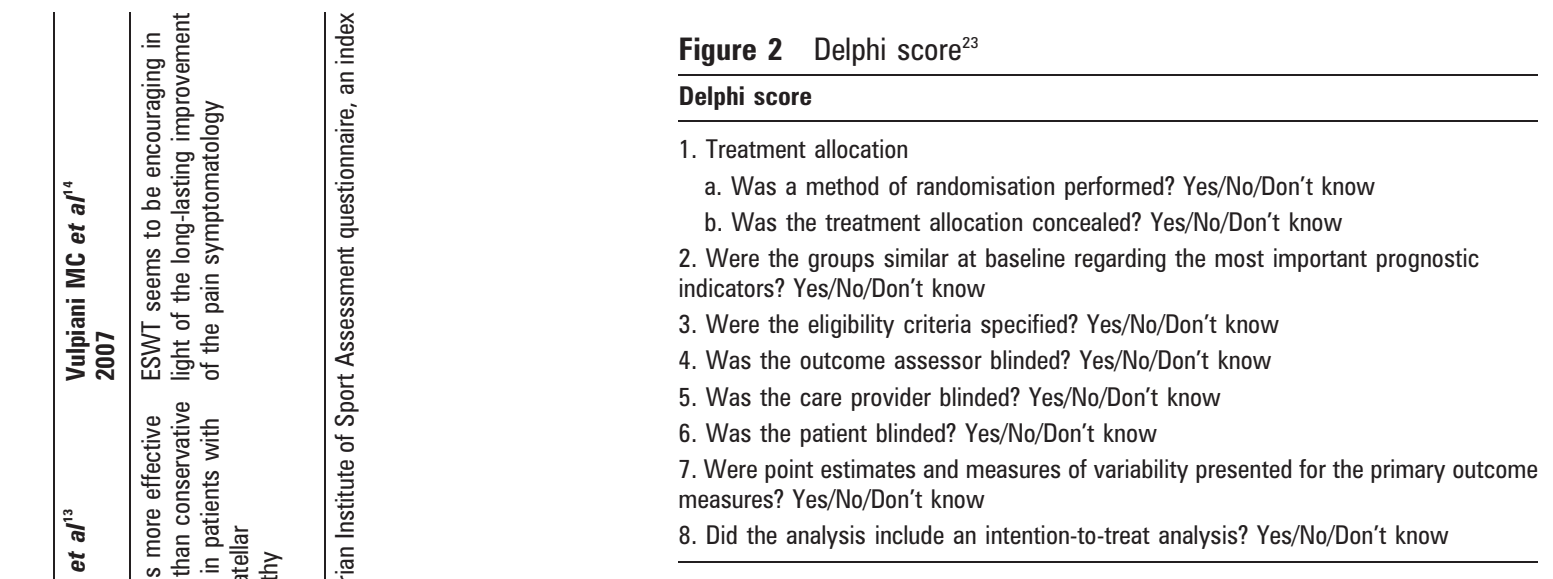

that patients with only mild complaints had a better prognosis and consequently influenced the results positively, but, because a strict randomisation was applied, patients with only mild complaints were found in both groups. Peers concluded that ESWT positively contributed to improvement of pain and function in the short-term treatment of patellar tendinopathy. ${ }^{26}$

In 2003 Taunton et al evaluated the effects of ESWT for patellar tendinopathy. In a randomised clinical trial, 10 patients in the study group received from three to five focused ESWT treatments with an energy level of $0.17 \mathrm{~mJ} / \mathrm{mm}^{2}$. In the control group, 10 patients underwent the same treatment procedure but with an absorbing pad between skin and probe, so only a placebo treatment was given. Evaluation up to 12 weeks after the last treatment included a questionnaire, VISA score and a vertical jump test. The VISA score and vertical jump test improved significantly in the study group. A limitation of this study was that no VAS score was used for the evaluation, even though it is a useful indicator of pain relief. Also in this study a short follow-up time was used and the number of patients was only 20. Taunton et al concluded that ESWT treatment could be of value as an additional treatment to other conservative therapies, like eccentric training. ${ }^{27}$

Wang et al (2007) evaluated the efficiency and safety of ESWT treatment for patellar tendinopathy. In a randomised controlled trial they compared one focused ESWT treatment of $0.18 \mathrm{~mJ} /$ $\mathrm{mm}^{2}$ with the results of conservative treatment. This study consisted of 27 patients ( 30 tendons) in the study group and 23 patients (24 tendons) in the control group. After 1, 3, 6, 12, 24 and 36 months VISA and VAS scores, functional improvement and ultrasonographic examination of the patellar tendon were evaluated. There was a significant improvement in patients who had been treated with ESWT. They showed functional improvement and both the VAS and the VISA scores improved. Ultrasonographic examination revealed a significant increase in vascularisation. There were no serious side effects or complications. According to Wang et al, this study had some limitations. The number of patients was small and the length of follow-up was relatively short, although the length of follow-up of this study was the largest of all seven studies included. The functional improvement of the knee was assessed subjectively on the performance of daily activities, including sports participation. Despite these limitations, they concluded that ESWT was more effective and safer than conservative treatment for patients with chronic patellar tendinopathy. ${ }^{13}$

In 2007 Vulpiani et al reported a prospective study on the treatment of jumper's knee using ESWT. All included patients had a confirmed jumper's knee for at least 3 months, with pain not responding to previous conservative treatment. All 73 
patients (83 tendons) received from three to five focused ESWT treatments with an energy level of $0.08-0.44 \mathrm{~mJ} / \mathrm{mm}^{2}$. Already 1 month after ESWT treatment a significant improvement in the average VAS score was found, and the VAS scale improved further during follow-up. Additional evaluations after 1 month and in short-term (6-12 months), medium-term (1324 months) and long-term (>24 months) periods showed satisfactory results in respectively $43 \%, 64 \%, 69 \%$ and $80 \%$ of the patients. However, 14 tendons were lost to follow-up. Clear limitations in this study were the study design, without a control group, and the impossibility of achieving a 100\% patient recall. The study of Vulpiani et al showed positive effects of ESWT treatment for patellar tendinopathy, so they concluded that the results were encouraging in light of the long-lasting improvement of the pain symptomatology. ${ }^{14}$

\section{DISCUSSION}

All of the seven studies included in this review concluded that ESWT seems to be an effective treatment for patellar tendinopathy. It is a safe treatment as well because no serious side effects have been reported. Since there are many differences in the treatment protocols used, it would be inappropriate to attempt a quantitative meta-analysis on the effectiveness of ESWT for patellar tendinopathy, but based on these studies it can be estimated that in approximately $74.7 \%$ of patients ESWT treatment resulted in improvement of pain and knee function. The results should be interpreted with caution, though, since the quality of the studies is variable (Delphi score ranged from 2 to 7 out of a maximum of 9). ${ }^{23}$ Three studies $^{61425}$ were nonrandomised, two studies had no control group, ${ }^{14}{ }^{25}$ and one study had a retrospective design. ${ }^{6}$ Of the RCTs, one study compared ESWT treatment with conservative treatment, ${ }^{13}$ while in others placebo ESWT was applied to the control group. ${ }^{23}{ }^{26}{ }^{27}$ One RCT was only single-blinded. ${ }^{24}$ Patients were blinded in only two studies. Further, in most studies small numbers of patients were used and the post-treatment followup time was generally short. ${ }^{6} 131424-27$ Because of the aforementioned limitations, which are also indicated by the fact that there were only two satisfactory Delphi scores, it is hard to draw firm conclusions about the effectiveness of ESWT for patellar tendinopathy.

Since no appropriate conservative or surgical treatments for this common injury have been described so far, we believe that the mainly positive results from these studies justify further research into the role that ESWT can play in the management of athletes with patellar tendinopathy. Double-blind, randomised, controlled studies in larger patient groups and with sufficient follow-up time, for at least 3 years, using standardised treatment protocols are necessary to assess the true value of ESWT for patellar tendinopathy. Based on the available evidence in the literature it is impossible to recommend a specific ESWT treatment protocol. Hence comparative studies between different treatment strategies are also urgently needed. In this way, important questions with regard to number and frequency of treatments, energy level and method of shockwave application will be answered. There is no consensus either about the use of anaesthetics; it seems that their use is unnecessary. Almost all patients in this review received ESWT therapy without anaesthetics and they all tolerated it well.

Research in the field of ESWT and tendinopathies is still hampered by the fact that both the pathophysiology of patellar tendinopathy and the exact working mechanism of ESWT have not been elucidated so far. The underlying pathology in patellar tendinopathy is considered to be a failed healing response due to a degenerative process resulting from excessive overload, ${ }^{813-15} 2125{ }^{27-30}$ rather than a prostaglandin-mediated inflammatory process. ${ }^{8}{ }^{10}$ Neovascularisation is often found in patellar tendinopathy, and it has been hypothesised that tendon pain is caused by sensory nerves that grow into the tendon, causing a neurogenic inflammation and pain response. ${ }^{30}$ A further explanation of the pain in patellar tendinopathy could be the disturbance of nociceptive transmission in the nervous system, by means of changed levels of substance P (SP), glutamate and tyrosine hydroxylase $(\mathrm{TH}) .{ }^{30}$ Little is known about the working mechanism of ESWT. In animal studies ${ }^{2}$ it has been demonstrated that ESWT may have an impact on the nociceptive transmission in the nervous system and that it can cause dysfunction of peripheral, sensory nerve fibres, resulting in pain relief. ${ }^{16}$ ESWT probably has an influence on neovascularisation as well. Surprisingly, Wang et al found, along with the clinical improvement of their patients, a significant increase of vascularisation after ESWT treatment. ${ }^{13}$ They supposed the increased vascularity led to better tissue regeneration in tendinopathies through a better blood supply. ${ }^{13}$ This finding is in flat contradiction to the aforementioned theory about neurovascular structures and pain. A possible explanation could be that, due to the improvement in pain and function, patients became more active, resulting in increased vascularity. ${ }^{31}$ In athletes the degree of neovascularisation can also vary during the season, ${ }^{32}$ but no data on sport participation were given by Wang. In order to develop an effective ESWT treatment protocol for patellar tendinopathy it is crucial to elucidate the exact pathophysiology of patellar tendinopathy and to clarify the working mechanism of shockwaves on (pathological) tendon tissue and neovascularisation. Without more basic knowledge of these phenomena, ESWT will remain a controversial treatment.

So far, ESWT has mainly been used to treat patients with a chronic severe patellar tendinopathy not responsive to other conservative treatments. The mean VISA score of the patients of the included studies was 47.8. In more than $50 \%$ of those studies, the mean duration of symptoms was over 6 months,

\section{What is already known on this topic}

- Patellar tendinopathy is a common chronic overuse injury of the patellar tendon based on failed tendon regeneration.

- Several conservative and surgical treatment options are used, but based on the present literature the most appropriate and effective treatment is unknown.

- ESWT is used for the conservative treatment of several chronic tendinopathies, including patellar tendinopathy.

\section{What this study adds}

Few studies are conducted into the effectiveness of ESWT for patellar tendinopathy with great variability in methodological quality.

- ESWT seems to be a safe and promising treatment for patellar tendinopathy.

- Further clinical and basic research into the effectiveness and working mechanism of ESWT in patellar tendinopathy is necessary. 
and patients had mostly had several other treatments already. This may have negatively influenced the outcome of the studies. We believe that ESWT could be of help in an earlier, less severe stage of the disease as well. ESWT can potentially play a role in the management of tendon pain and therefore should be incorporated into a more comprehensive exercise-based rehabilitation programme. ${ }^{15}{ }^{33}$ A more comprehensive programme also seems justified when we look at the VISA scores after treatment. Healthy athletes have a VISA score of around 95 points. In these studies the maximum VISA score was 92 and the average VISA score was 78. Peers et al showed that results of ESWT therapy equal the results of surgical treatment for patellar tendinopathy; results showed a VISA score of respectively 83 and $70 .{ }^{6}$ Bahr et al compared surgical treatment with eccentric training; results showed a VISA score of 70 for both groups. ${ }^{4}$ ESWT treatment seems just as effective as, or even more effective than, existing treatments for patellar tendinopathy, but a completely normal knee function is probably not feasible with monotherapy. A more comprehensive exercisebased rehabilitation programme could therefore help to achieve higher VISA scores.

In conclusion, ESWT seems to be a safe and promising treatment for patellar tendinopathy. Because ESWT treatment seems to have a positive effect on pain and function, it could be part of a rehabilitation programme for this chronic overuse injury. However, based on the current knowledge it is impossible to recommend a specific treatment protocol. Further basic and clinical research into the working mechanism and effectiveness of ESWT for patellar tendinopathy is necessary.

\section{Competing interests: None.}

\section{REFERENCES}

1. Blazina ME, Kerlan RK, Jobe FW. Jumper's knee. Orthop Clin North Am 1973:4:665-78.

2. Lian $\mathbf{O B}$, Engebretsen L, Bahr R. Prevalence of jumper's knee among elite athletes from different sports: a cross sectional study. Am J Sports Med 2005:33:561-7.

3. Zwerver J, Bredeweg SW. Prevalence of jumper's knee among non-elite athletes from three different sports. Abstract book of the XXIX FIMS World Congress of Sports Medicine, Beijing, 2006:273.

4. Bahr R, Fossant B, Løken S, et al. Surgical treatment compared with eccentric training for patellar tendinopathy (Jumper's Knee). A randomized, controlled trial. J Bone Joint Surg Am 2006;88:1689-98.

5. Kettunen JA, Kvist M, Alanen E, et al. Long-term prognosis for jumper's knee in male athletes. A prospective follow-up study. Am J Sports Med 2002;30:689-692.

6. Peers KHE, Lysens RJJ, Brys P, et al. Cross-sectional outcome analysis of athletes with chronic patellar tendinopathy treated surgically and by extracorporeal shock wave therapy. Clin J Sport Med 2003;13:79-83
7. Visnes $\mathbf{H}$, Bahr R. The evolution of eccentric training as treatment for patellar tendinopathy (jumper's knee) - a critical review of exercise programs. $\mathrm{Br} J$ Sports Med 2007:41:217-23.

8. Alfredson $\mathbf{H}$. The chronic painful achilles and patellar tendon: research on basic biology and treatment. Scan J Med Sci Sports 2005;15:252-9.

9. Kannus P. Etiology and pathophysiology of chronic tendon disorders in sports Scand J Med Sci Sports 1997:7:78-85.

10. Khan KM, Cook JL, Kannus P, et al. Time to abandon the "tendinitis myth". BMJ 2002:324:626-7.

11. Cook JL, Khan KM. What is the most appropriate treatment for patellar tendinopathy? Br J Sports Med 2001;35:291-4.

12. Peers KHE, Lysens RJJ. Patellar tendinopathy in athletes; current diagnostic and therapeutic recommendations. Sports Med 2005;35:71-87.

13. Wang CJ, Ko JY, Chan YS, et al. Extracorporeal shockwave for chronic patellar tendinopathy. Am J Sports Med 2007:35:972-8.

14. Vulpiani MC, Vetrano M, Savoia W, et al. Jumper's knee treatment with extracorporeal shock wave therapy: a long-term follow-up observational study. J Sports Med Phys Fitness 2007:47:323-8.

15. Kontouris A, Cook J. Rehabilitation of Achilles and patellar tendinopathies. Best Pract Res Clin Rheumatol 2007:21:295-316. Review.

16. Chung B, Wiley JP. Extracorporeal shockwave therapy. Sports Med 2002;32:851-65

17. Ogden JA, Tóth-Kischkat A, Reiner S. Principles of shock wave therapy. Clin Orthop Relat Res 2001;387:8-17.

18. Peers KH. ESWT. Proefschrift. KU Leuven, 2003.

19. Furia JP, Rompe JD. Extracorporeal shock wave therapy in the treatment of chronic plantar fasciitis and Achilles tendinopathy. Curr Opin Orthop 2007:18:102-11.

20. Sems A, Dimeff R, lannotti JP. Extracorporeal shock wave therapy in the treatment of chronic tendinopathies. J Am Acad Orthop Surg 2006;14:195-204.

21. Hsu RWW, Hsu WH, Tai CL, et al. Effect of shock-wave therapy on patellar tendinopathy in a rabbit model. J Orthop Res 2004;22:221-7.

22. Maier M, Averbeck B, Milz S, et al. Substance P and prostaglandin E2 release after shock wave application to the rabbit femur. Clin Orthop Relat Res 2003:406:237-45.

23. Verhagen AP, de Vet HCW, de Bie RA, et al. The Delphi list: a criteria list for quality assessment of randomized clinical trials for conducting systematic reviews developed by Delphi consensus. J Clin Epidemiol 1998;51:1235-41.

24. Vara F, Garzon N, Ortega N. Treatment of the patellar tendinitis with local application of extracorporeal shock waves. Abstract from the 4th Congress of the International Society for Musculoskeletal Shock Wave Therapy. Naples, 2000.

25. Lohrer H, Schöll J, Arentz S. Achillodynie und patellaspitzensyndrom - Ergebnisse der behandlung austherapierter, chronischer fälle mit radialen sto $\beta$ wellen. Sportverletz. Sportschaden 2002;16:108-14.

26. Peers KH. Extracorporeal Shock Wave Therapy in chronic patellar tendinopathy: a randomised double-blinded, placebo-controlled trial. Proefschrift. KU Leuven, 2003.

27. Taunton KM, Taunton JE, Khan KM. Treatment of patellar tendinopathy with extracorporeal shock wave therapy. BC Medical Journal 2003;45:500-7.

28. Tasto JP, Cummings J, Medlock V, et al. The tendon treatment center: new horizons in the treatment of tendinosis. Arthroscopy 2003;19:213-23.

29. Peers KH. Chronic tendinopathy. Proefschrift. KU Leuven, 2003

30. Lian Ø, Dahl J, Ackermann PW, et al. Pronociceptive and antinociceptive neuromediators in patellar tendinopathy. Am J Sports Med 2006;34:1801-8.

31. Boesen MI, Koening MJ, Torp-Pedersen, et al. Tendinopathy and Doppler activity: the vascular response of the Achilles tendon to exercise. Scand J Med Sci Sports 2006:16:463-9.

32. Malliara $\mathbf{P}$, Cook J. Patellar tendons with normal imaging and pain: change in imaging and pain status over a volleyball season. Clin J Sport Med 2006;16:388-91.

33. Alfredson $\mathbf{H}$, Cook J. A treatment algorithm for managing Achilles tendinopathy: new treatment options. Br J Sports Med 2007;41:211-16.

34. Visentini PJ, Khan KM, Dook JL, et al. The VISA score: an index of severity of symptoms in patients with jumper's knee (patellar tendinosis). Victorian Institute of Sports Tendon Study Group. J Sci Med Sport 1998;1:22-8. 